\title{
Buttonhole: tutto il contrario di quello che ho sempre fatto
}

\author{
Hilary Riva ${ }^{1}$, Chiara Dossi ${ }^{2}$, Giuseppe Bonforte ${ }^{2}$ \\ ${ }^{1}$ Scuola di Specialità Nefrologia e Dialisi, Università degli Studi Milano Bicocca, Milano \\ ${ }^{2}$ Nefrologia e Dialisi Azienda Ospedaliera Sant'Anna, Como
}

\begin{abstract}
BUTTONHOLE: THE OPPOSITE OF WHAT I'VE ALWAYS DONE
Abstract. The buttonhole technique (BHT) represents an arteriovenous fistula (AVF) puncture tool that allows positioning the needle always at the same site of either the left or right arm of hemodialysis patients. This procedure is more comfortable for patients, and reduces hemostasis time and aneurysmatic degradation of AVF. Despite BHT been rapidly accepted by patients and nurses, some physicians are still sceptical. BHT requires paying particular attention in managing subcutaneous tunnellization and preserve it for each of the next incannulations. Physicians and nurses need continuous training to minimize infective and/or traumatic complications. BHT should be suggested for each hemodialysis patient with puzzling venipuncture.
\end{abstract}

Key words: Vascular access, Arterovenous fistula cannulation, Buttonhole, Hemodialysis

Conflict of interest: None.

Ricevuto: 19 Dicembre 2012; Accettato: 2 Maggio 2013

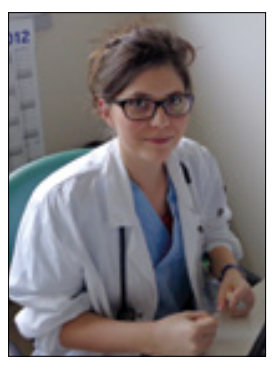

Hilary Riva
La buttonhole technique (BHT) è una tecnica di puntura della fistola arterovenosa (FAV) che prevede l'inserimento dell'ago sempre nella stessa sede, con il medesimo angolo di ingresso e alla stessa profondità.

La BHT ha avuto, finora, un ruolo marginale nella pratica clinica e, nonostante la prima pubblicazione di Twardowski risalga al ' $79(1,2)$, questa tecnica è tornata alla luce solo nell'ultimo decennio, grazie al forte entusiasmo dei pazienti e, soprattutto, grazie alla spinta del personale infermieristico; la maggior parte della letteratura al riguardo proviene, infatti, da riviste infermieristiche (3-6).

A livello teorico, la BHT è una scelta che appare irrazionale; per comprenderla forse è utile conoscere la sua storia. La nascita della BHT si deve a un'infermiera, Suor Helena Kubara, caposala che lavorava al fianco del Dr. Twardowski in un centro di dialisi in Polonia. Nel centro in questione, siamo agli inizi degli anni '70; c'erano a disposizione aghi di buona fattura, quindi più taglienti, importati da Seattle, e aghi di minore qualità, prodotti in Polonia, che, venendo anche riutilizzati, diventavano sempre meno taglienti. Ai tempi, la caposala seguiva un paziente con una fistola complessa, pungibile solo in un breve tratto, che l'ha obbligata a riposizionare gli aghi sempre nei medesimi due punti di inserimento. Così, la nascita della BHT è stata la conseguenza di un insieme di eventi fortuiti: la necessità di pungere sempre lo stesso punto, la scarsità di risorse che ha obbligato il personale a riutilizzare aghi già poco taglienti e la presenza di un'infermiera con forte spirito critico che ha fatto utili osservazioni e considerazioni, con le quali ha salvaguardato la FAV del primo paziente per ben 26 anni. Gli inglesi definirebbero questa successione di eventi con il termine serendipity: un evento fortuito, qualcosa di mai cercato ma trovato per caso.

Nel centro di dialisi di Twardowski, la BHT acquistò grande popolarità tra i pazienti e si diffuse rapidamente. Dalle prime pubblicazioni la tecnica sembrava molto promettente (Tab. I) e offriva numerosi vantaggi rispetto alla puntura a sito variabile e la sola complicanza segnalata da Twardowski era l'aumentato rischio di infezione in sede di puntura.

Malgrado ciò, nel resto del mondo la BHT ha incontrato molta resistenza che ha limitato la sua diffusione.

Finora, il più grande ostacolo all'adozione della BHT è stata la comune convinzione che la migliore tecnica di puntura sia la tecnica "a scala di corda", con la conseguente demonizzazione della puntura "ad area", erroneamente confusa con la BHT. La BHT è stata, quindi, guardata con molto sospetto, perché chiedeva agli operatori di pungere la fistola seguendo delle regole contrarie a quelle applicate fino a quel momento, anche se rimaneva la prova provata che, in certe condizioni, si può pungere una fistola sempre nello stesso punto (Fig. 1). Uno dei motivi dello scarso sviluppo della BHT è dovuto al fatto che molti pensano di sapere a priori come si applica 


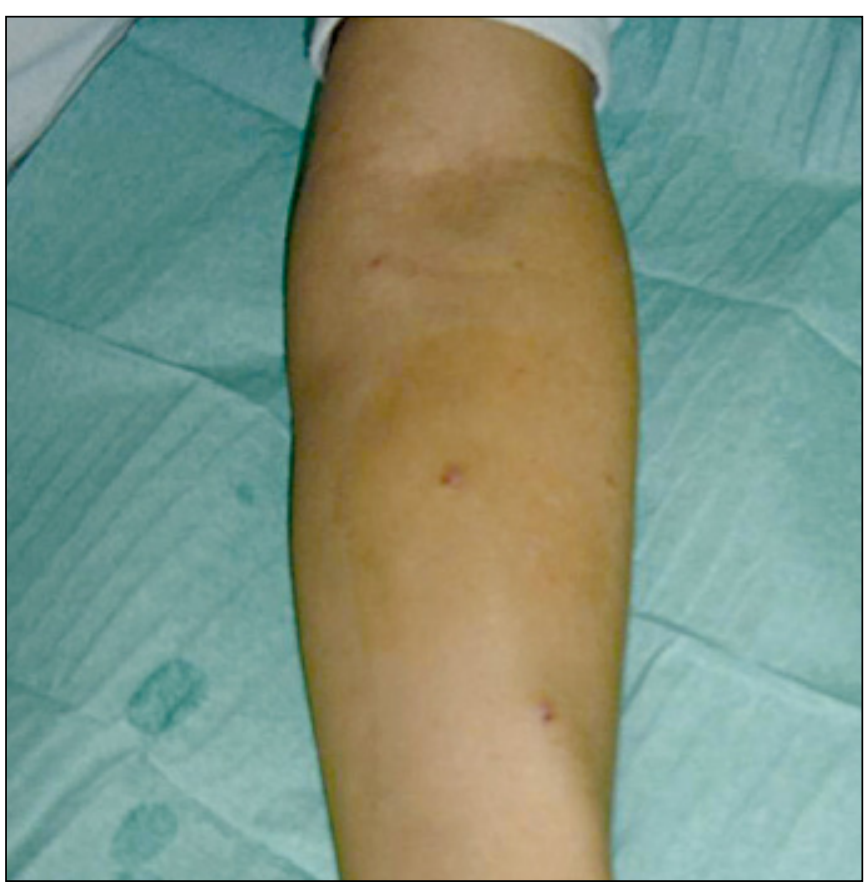

Fig. 1 - Fistola arterovenosa punta con la tecnica buttonhole.

la tecnica, senza mai confrontarsi con il protocollo descritto da Twardowski.

Tale protocollo prevede la selezione sul decorso della FAV di punti idonei per praticare l'incannulazione, da effettuare tenendo l'ago inclinato a $25-35^{\circ}$. Le volte successive è fondamentale l'attenta disinfezione e la rimozione dell'intera crosta, utilizzando un piccolo ago per cercare di sollevarla tutta intera (Fig. 2). Si disinfetta nuovamente e si procede all'incannulazione nel punto in cui vi era la crosta, utilizzando un ago tagliente da dialisi e cercando di mantenere la stessa inclinazione del tunnel già formato. In genere, dopo circa 6-8 incannulazioni, fatte possibilmente sempre dallo stesso operatore con un ago tagliente, il tunnel cutaneo è delimitato ed è, quindi, possibile iniziare a utilizzare gli aghi smussi.

Uno dei punti critici per l'utilizzo della BHT è la presenza continuativa sempre dello stesso operatore per allestire il

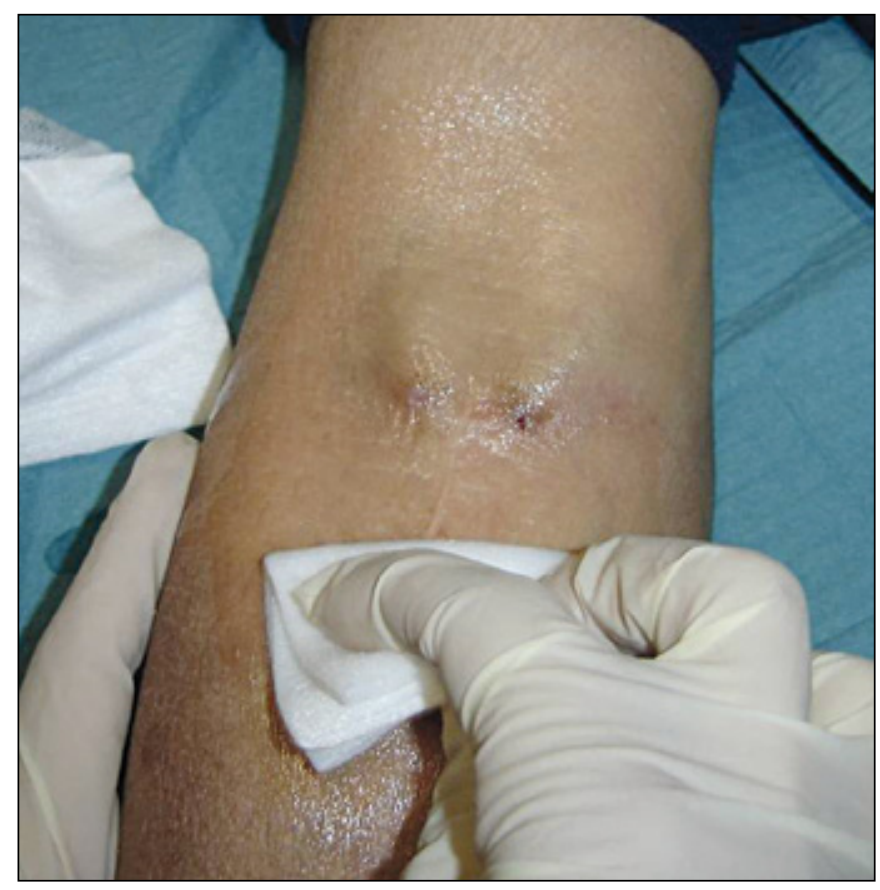

Fig. 2 - Immagine del buttonhole con la crosta (destra) e dopo la sua rimozione (sinistra).

tunnel sottocutaneo, ragione per la quale, da qualche anno, è in corso la ricerca di nuovi metodi per favorire la sua creazione, sfruttando device appositamente studiati che si propongono come scorciatoia per la formazione del buttonhole. Al momento, in letteratura sono stati descritti alcuni dispositivi creati per essere lasciati nel sito di puntura: uno é in policarbonato (8), a forma di puntina da disegno, mentre l'altro è un catetere in poliuretano (9). Queste tecniche sono state sperimentate su casistiche limitate di pazienti ed è ancora oggi dibattuta la loro reale utilità; il metodo per ora più valido per la creazione del tunnel resta il mantenimento dello stesso operatore.

La vera complicanza di questa tecnica è l'infezione $(10,11)$. Per evitarla, bisogna aumentare le misure di asepsi, seguire rigidamente il protocollo e selezionare accuratamente i pazienti. I maggiori fattori di rischio di infezione sono la formazione di un falso tunnel, l'incompleta rimozione della crosta

TABELLA I - VANTAGGI DELLE DIVERSE TECNICHE DI PUNTURA DELLA FAV (7)

\section{Puntura a sito variabile}

- non necessario sempre lo stesso operatore

- non richiede ulteriore formazione

- minore costo dei materiali (no kit aghi smussi)

- rimozione di croste non necessaria, quindi:

. irrilevante la compliance del paziente

minor rischio di complicanze infettive
Puntura a sito costante (BHT)

- riduzione del dolore con conseguente maggiore sopportabilità

del paziente e minore ansia del paziente e del personale

- maggiore facilità di incannulazione

- minor tempo necessario per l'attacco (non necessaria la ricerca

del sito di puntura)

- riduzione degli aneurismi/arresto della loro evoluzione

- possibilità di autoincannulamento

- riduzione del tempo necessario per l'emostasi 
e la presenza di una carica batterica maggiore sulla cute dei pazienti emodializzati. Le segnalazioni delle complicanze infettive presenti in letteratura descrivono eventi di diversa gravità, che vanno dalla semplice infezione locale a casi di setticemia (12).

I fattori di rischio indicati sono in buona parte modificabili con la formazione e la motivazione di tutto il personale infermieristico: emblematica è l'esperienza di un centro di dialisi in Belgio, nel quale si è vista un'importante riduzione delle complicanze infettive in seguito a un adeguato retraining dello staff infermieristico (13).

Infine non va trascurata la selezione del paziente: pazienti poco complianti e/o con una scarsa propensione all'igiene personale (che può, anzi, deve essere insegnata a priori) e verosimilmente quelli immunodepressi sono inadatti per questo tipo di puntura.

La BHT è, quindi, una tecnica valida, apprezzata sia dai pazienti che da una buona parte dello staff medico-infermieristico, sicuramente in grado di offrire importanti vantaggi, ma le cui complicanze non sono trascurabili e sono legate principalmente al rischio infettivo.

La BHT è, in definitiva, una tecnica di puntura della fistola che non può essere applicata in tutti i pazienti, ma che offre notevoli benefici in pazienti selezionati, quando il personale medico e infermieristico è adeguatamente formato e motivato.

\section{Riassunto}

La buttonhole technique (BHT) è un metodo di puntura della fistola arterovenosa (FAV), che prevede il posizionamento degli aghi sempre esattamente nello stesso punto. Questa tecnica, per alcuni aspetti controversa, ha diversi vantaggi: rende più sopportabile l'incannulazione, accorcia i tempi dell'emostasi e riduce la degenerazione aneurismatica della FAV. Per questo è stata rapidamente ben accettata dai pazienti e dal personale infermieristico, anche se permane lo scetticismo di alcuni medici. La spiegazione di tale posizione sta nel dover pensare e fare tutto il contrario di quanto detto e fatto precedentemente. Forse, conoscere la storia di come è nata questa tecnica può aiutare a rimuovere alcuni dubbi. La BHT è una tecnica che non si può improvvisare e che richiede dedizione sia nelle fasi iniziali, per creare il tunnel sottocutaneo, sia nelle fasi successive ad ogni incannulazione, per conservarlo integro. Non è da sottovalutare il rischio di infezione insito nell'applicazione di questa procedura che solo un corretto e continuo training dell'equipe può prevenire o intercettare. La BHT non può essere proposta indiscriminatamente a tutti i pazienti sottoposti a emodialisi, ma va riservata alle FAV complesse dove la normale puntura risulta complicata e complicante.

Parole Chiave: Accesso vascolare, Puntura della FAV, Puntura a sito costante, Emodialisi

Dichiarazione di conflitto di interessi: Gli Autori dichiarano di non avere conflitto di interessi

Indirizzo degli Autori:

Dr. Giuseppe Bonforte

U.O. Nefrologia e Dialisi Ospedale S. Anna

Via Ravona 20

22020 San Fermo della Battaglia (CO)

giuseppe.bonforte@hsacomo.org

\section{Bibliografia}

1. Twardowski Z, Lebek R, Kubara H. [6-years experience with the creation and use of internal arteriovenous fiostulae in patients treated with repeated hemodialysis]. Pol Arch Med Wewn 1977; 57 (3): 205-14.

2. Twardowski Z. Constant site (buttonhole) method of needle insertion for hemodialysis. Dial Transpl 1995; 24: 559-62.

3. MacRae JM. A randomized trial comparing buttonhole with rope ladder needling in conventional hemodialysis patients. Clin J Am Soc Nephrol 2012; 7 (10): 1632-8.

4. Pergolotti A. The effect of the buttonhole method vs. the traditional method of AV fistula cannulation on hemostasis, needle stick pain, pre-needle stick anxiety, and presence of aneurysms in ambulatory patients on hemodialysis. Nephrol Nurs J 2011; 38 (4): 333-6.

5. Mott S, Moore H. Kinder, gentler methods for scab removal in buttonhole access. Nephrol Nurs J 2011; 38 (5): 439-43.

6. Ball LK. The buttonhole technique: strategies to reduce infections. Nephrol Nurs J 2010; 37 (5): 473-7.
7. Kim MK. Clinical effects of buttonhole cannulation method on hemodialysis patients. Hemodial Int 2013; 17 (2): 294-9.

8. Donnelly SM. Use of the BioHole ${ }^{\mathrm{TM}}$ device for the creation of tunnel tracks for buttonhole cannulation of fistula for hemodialysis. Hemodial Int 2011; 15 (2): 243-9.

9. Donnelly SM, Marticorena RM, Hunter J, Goldstein MB. Supercath Safety Clampcath buttonhole creation: Complication of catheter breakage. Hemodial Int 2012.

10. Nesrallah GE. Staphylococcus aureus bacteremia and buttonhole cannulation: long-term safety and efficacy of mupirocin prophylaxis. Clin J Am Soc Nephrol 2010; 5: 1047-53.

11. van Loon MM. Buttonhole needling of haemodialysis arteriovenous fistulae results in less complications and interventions compared to the rope-ladder technique. Nephrol Dial Transplant 2010; 25 (1): 225-30.

12. Gray N. The risk of sepsis from buttonhole needling must be appreciated. Nephrol Dial Transplant 2010; 25 (7): 2385-6.

13. Labriola L. Infectious complications following conversion to buttonhole cannulation of native arteriovenous fistulas: a quality improvement report. Am J Kidney Dis 2011; 57 (3): 442-8. 